\title{
Avalanches and the Renormalization Group for Pinned Charge-Density Waves
}

\author{
Onuttom Narayan \\ Department of Physics, Harvard University, Cambridge, MA 02138 \\ and \\ ATET Bell Laboratories, 600 Mountain Avenue, Murray Hill, NJ 07974 \\ A. Alan Middleton \\ NEC Research Institute, 4 Independence Way, Princeton, NJ 08540
}

(May 12th, 1993)

\begin{abstract}
The critical behavior of charge-density waves (CDWs) in the pinned phase is studied for applied fields increasing toward the threshold field, using recently developed renormalization group techniques and simulations of automaton models. Despite the existence of many metastable states in the pinned state of the CDW, the renormalization group treatment can be used successfully to find the divergences in the polarization and the correlation length, and, to first order in an $\epsilon=4-d$ expansion, the diverging time scale. The automaton models studied are a charge-density wave model and a "sandpile" model with periodic boundary conditions; these models are found to have the same critical behavior, associated with diverging avalanche sizes. The numerical results for the polarization and the diverging length and time scales in dimensions $d=2,3$ are in agreement with the analytical treatment. These results clarify the connections between the behaviour above and below threshold: the characteristic correlation lengths on both sides of the transition diverge
\end{abstract}


with different exponents. The scaling of the distribution of avalanches on the approach to threshold is found to be different for automaton and continuousvariable models.

Typeset using REVTEX 


\section{INTRODUCTION}

The static and dynamic behaviour of sliding charge density waves (CDWs) is perhaps the most well studied example of a class of problems involving the transport of an elastic medium through a disordered background. The CDW, which behaves like an elastic medium, is pinned by impurities distributed randomly throughout the material. As the magnitude of an externally applied electric field is varied, a depinning transition is seen, from a stationary phase at weak fields, to a moving phase at strong fields where the CDW slides through the material. In the vicinity of this depinning transition, the dynamics of the CDW are correlated over long distances, with characteristic correlation lengths diverging at the threshold field. It has been shown 1 that the behaviour near the threshold field can be studied as a critical phenomenon associated with a second order phase transition.

Extensive numerical simulations $\mathrm{B}^{\mathrm{B}}$ on a number of classical models for CDWs have helped in understanding the critical properties in the vicinity of the depinning transition. In particular, critical exponents describing the scaling of physical quantities can be determined for various spatial dimensions of the CDW. Recently, it has also proved possible to obtain analytically the behaviour in the moving phase above threshold, through a renormalization group treatment 9 The results, obtained within an $\epsilon$-expansion for $d=4-\epsilon$ spatial dimensions, agree very well with the numerical simulations.

While the critical behaviour above threshold is fairly well understood, it is more difficult to carry out an analytical treatment below threshold. The reason why the analysis is simpler in the moving phase is that, for models in which the elastic interactions within the CDW are strictly convex, and no dislocations ("phase slips") are allowed, the system approaches a unique steady-state configuration at long times (up to time translations). 10 A perturbation expansion around some relatively simple configuration is thus more likely to succeed. In the stationary phase, on the other hand, the impurities can pin the CDW in any of several different states; the particular state that the CDW reaches depends on the past history of the system. Any analytical treatment must include this history dependence, instead of being 
the simple search for a single attractor, as is sufficient above threshold.

The history dependence of the CDW below threshold is most easily seen in the polarizability. If the driving force $F$ (produced by the external electric field) is raised monotonically towards the depinning threshold $F_{T}$, the response $\chi^{\uparrow}(F)$ to infinitesimal increases in the force becomes larger and larger, and diverges at $F_{T}$ as $\chi^{\uparrow}(F) \sim\left(F_{T}-F\right)^{-\gamma}$, with an exponent $\gamma$. Also, at any value of $F$, the response of the CDW to increasing the force further is dominated by localized regions of activity, or "avalanches", whose characteristic size can be used to define a diverging correlation length $\xi \sim\left(F_{T}-F\right)^{-\nu}$. If instead of this monotonic approach, the force is lowered from just below threshold, the polarizability $\chi^{\downarrow}(F)$ does not diverge at $F_{T}$ (although, depending on the specific model used for the CDW, it may have a cusp singularity). It is thus necessary to distinguish between the upwards polarizability, $\chi^{\uparrow}(F)$, and the downwards polarizability $\chi^{\downarrow}(F)$.

In this paper, we show how it is possible to obtain analytically the critical behaviour for the 'natural' monotonic approach to threshold, through an expansion around the mean field solution with the proper history dependence. Our results are in good agreement with recent numerical simulations, as well as new data that we present here. We find that the correlation length exponent is given by

$$
\nu=2 / d
$$

for a $d$ dimensional system, while the polarizability exponent is

$$
\gamma=4 / d
$$

These exponents can in fact be obtained for a highly simplified CDW model 11 that can be solved exactly. However, the dynamic exponent z cannot be obtained from this model: we show that $z$ is equal to its value above threshold

$$
z=2-\epsilon / 3+O\left(\epsilon^{2}\right)
$$

in $d=4-\epsilon$ dimensions. Later in this paper, we discuss how the model of Ref. 11 corresponds to a pathological limit, and why, despite this, it obtains $\nu$ and $\gamma$ correctly. 
The solution that we obtain is similar in form to the solution above threshold. 9 However, there are complications with two-sided scaling. This is because, as was seen above threshold, there is an extra relevant operator at the fixed point of the renormalization group, which affects only the statics and not the dynamics. This operator has very different consequences in the pinned and moving phases of the CDW, resulting in different correlation length exponents on the two sides of the phase transition. The absence of two-sided scaling is unlike the behaviour for phase transitions in equilibrium systems, and conventional arguments about the properties of scaling functions must be used with caution here.

In "phase-only" models for CDWs, the CDW is described by a periodic modulation in the density of electrons in the material; the phase of this modulation is assumed to be the only dynamical variable of importance and is assumed to be a continuous function of position. In numerical simulations, the system is discretized to a lattice, with a continuous phase variable at each site. Although simulations on such continuous variable models have been performed 6 , it is much more efficient to simplify the model, with the phase of the CDW at any lattice site restricted to being an integer multiple of $2 \pi$ away from a (site dependent) preferred phase, and time being incremented in discrete steps. 0.8 The resulting automaton models, which differ in details of how these approximations are made, yield far more precise results. Within the numerical uncertainties, much of the critical behaviour is the same for both classes of models. In particular, the correlation length exponent $\nu$ and (with larger numerical uncertainties) the polarizability exponent $\gamma$ appear to be the same. An important exception to this is the downward polarizability, $\chi^{\downarrow}$ : as already mentioned, the nature (or even the existence) of a cusp singularity in $\chi^{\downarrow}$ does depend on the details of the model used, and is in fact even different in different continuous variable models. However, this difference is not seen in the monotonic approach, which is what we shall be interested in here.

Since the approach to threshold causes the system to become increasingly unstable, with larger and larger avalanches and a divergent polarizability, it is perhaps not surprising that differences between automaton and continuous dynamics do not affect the critical behaviour. An important exception to this rule is the distribution for the number of avalanches. For 
continuous dynamics, an infinitesimal increase in $F$ triggers avalanches in which the change in the phase at any point is bounded above by $2 \pi$ (corresponding to unity in the automaton models). Most of the avalanche area advances by almost $2 \pi$. In large avalanches, it is highly probable that a subsequent small increase in $F$ will "retrigger" avalanches in the same region, as the original unstable point is likely to be nearly unstable at the completion of the first avalanche. Avalanches of usually decreasing size will be retriggered until the region is more stable. In the automaton models, owing to the discretization of the CDW phases, several "retriggered" avalanches are grouped together into a single large avalanche. This difference between the two classes of models is one that involves the behaviour at short times; this does not alter long-wavelength low-frequency characteristics that determine $\nu$ and $z$ (nor $\gamma$, which involves a spatial average over the entire system). However, it may affect avalanche distributions, which do depend on how avalanches are grouped. In this paper we present numerical data on the distribution of avalanches for the automaton models, and examine the differences with results for continuous variable models.

The automaton models for CDWs are closely related to some of the sandpile models that have been proposed and studied recently. 12 We find that the avalanche distribution that we obtain here for the CDW automaton models agrees very well with the numerical results on sandpiles at the critical point 13 The dynamic exponent, $z$, that relates the characteristic duration of an avalanche to its size, is also found to be the same numerically for both classes of models:

$$
\begin{aligned}
& z(d=2)=1.32 \pm 0.04 \\
& z(d=3)=1.65 \pm 0.06
\end{aligned}
$$

The approach to threshold for sandpile models has been treated through numerics and scaling arguments by Tang and Bak1 14 ; our results differ in certain respects from theirs, which we discuss in detail later in this paper.

The connection between sandpiles and CDW models can be exploited to obtain the dynamic exponent $z$ for two dimensional systems. Majumdar and Dhar 15 have shown that 
this exponent is equal to $5 / 4$ for sandpile models in $d=2$; this is in fair agreement with

the numerical results for CDWs.16 They are also able to relate the two exponents that enter the avalanche distribution to each other, thus leaving only one of the two to be determined numerically. In this paper we show that it is in fact possible to obtain the same exponent identity from completely different scaling arguments on CDWs, if we assume that the total rate of avalanche generation (which is dominated by small avalanches) is not singular as threshold is approached. (We have verified this assumption numerically for the automaton models.)

The rest of this paper is organized in the following manner. Section II carries out the analytical treatment of the behaviour below threshold, and obtains the critical exponents. Section III examines the numerical results, which agree quite well with the analytical predictions. Section IV compares the distribution of avalanches for CDW continuous dynamics, CDW automata, and sandpile models, as well as exploring other connections to sandpile models. Section V discusses the relationship of this work to earlier results, and its possible relevance to other physical systems.

\section{ANALYTICAL RESULTS.}

The model that we use in this section is the Fukuyama-Lee-Rice model, 17 which assumes that the distortions of the CDW are continuous (i.e. that there are no dislocations). The dynamics can then be expressed in terms of a phase variable, $\phi(x ; t)$, which measures the distortions with respect to an ideal undistorted CDW. (The position $x$ is a $d$-dimensional vector.) Assuming that the dynamics are strongly overdamped, and are given by a simple relaxation of an energy functional $H$, the equation of motion is

$$
\partial \phi(x ; t) / \partial t=-\partial H / \partial \phi=\nabla^{2} \phi(x ; t)+F+h(x) Y(\phi-\beta(x)) .
$$

In Eq.(5) there are three terms on the right hand side: a simple elastic force that arises from an elastic energy $\frac{1}{2}(\nabla \phi)^{2}$, a uniform force $F$ from the external electric field, and a force 
$h(x) Y(\phi-\beta(x))$ from the impurity pinning. This impurity force has an explicit dependence on the position $x$, arising from the quenched randomness in the location of the impurities, through the functions $h(x)$ and $\beta(x)$. These correspond physically to the strength of the impurity pinning, $h(x)$, and a preferred phase $\beta(x)$ selected by the impurities. (In order to fix the normalization of $h$, we choose $|Y|$ to have a maximum value of 1.) Since the impurities have only short range correlations, $h(x)$ and $\beta(x)$ are taken to be uncorrelated from one position to another, with a distribution $\rho(h)$ for $h$ and a uniform distribution over $[0,2 \pi)$ for $\beta$. The pinning force is the derivative, $-\partial_{\phi} V(\phi ; x)$ of an impurity pinning potential. Because of the $2 \pi$ translational invariance of the CDW, the force $Y$ has to be $2 \pi$-periodic in $\phi$.

Among the quantities of physical interest in this model below threshold are the polarization, $P$, defined as the mean displacement from some initial configuration of the phase $\phi$; close to threshold, the singular part of $P$ scales as

$$
P_{s}(F)=\left[\langle\phi\rangle-\langle\phi\rangle_{\text {initial }}\right]_{s} \sim\left(F_{T}-F\right)^{-\gamma+1} .
$$

The derivative of this with respect to $F$ is the upwards polarizability, defined earlier as the response to an infinitesimal increase in $F$, which scales as

$$
\chi^{\uparrow}(F) \equiv \lim _{\Delta F \rightarrow 0^{+}}[P(F+\Delta F)-P(F)] / \Delta F \sim\left(F_{T}-F\right)^{-\gamma} .
$$

In Ref. 9, the behaviour above threshold for models of this type was analyzed. This was done by expanding around the solution of Eq.(5) within a mean field approximation, which is obtained by replacing the short-ranged elastic term $\nabla^{2} \phi$ with an infinite-ranged interaction, $\langle\phi\rangle-\phi$. The mean field solution is known to depend on the details of the pinning potential $V(\phi)$ : for instance, the velocity above threshold scales as $v \sim\left(F-F_{T}\right)^{\beta}$ in the critical regime, 1 with an exponent $\beta_{M F}=3 / 2$ for smooth pinning potentials $\beta_{M F}=1$ for pinning potentials which have wedge-shaped linear cusped maxima. of universality arises from the fact that, for smooth pinning potentials, when the phase $\phi$ at any site passes through an unstable point in its local effective potential, it spends a long 
time accelerating before jumping forward to a new stable state. This results in a second time scale that diverges at threshold (in addition to the natural scale of $2 \pi / v$ ). No such second divergent time scale is present for linear cusped potentials, for which the acceleration time remains of $O(1)$ arbitrarily close to threshold. Despite this difference in behaviour for the two types of potentials in mean field theory, numerically it is found that the critical behaviour is independent of the shape of the pinning potential for $d<4$, and is in fact even the same for automaton models. In Ref. 9, it was argued that this is because the dynamics for small $d$ are very irregular: when the phase at any site jumps forward, it produces a sharp force on all the neighbours to which it is elastically coupled. For large $d$, the total elastic force acting on any phase is the sum of contributions from a large number of neighbours; however, as $d$ is decreased, the elastic force becomes more and more jerky. For sufficiently small $d$, the phase $\phi$ of the CDW at any site is 'kicked' by its neighbours over the maximum of the local effective potential it experiences. The details of the pinning potential are no longer important, and the second divergent time scale is eliminated, restoring universality. The expansion around mean field theory was accordingly carried out for linear cusped potentials, for which the second time scale is absent initially, so that the expansion is better behaved.

When $F_{T}$ is approached monotonically from below threshold, the critical behaviour is controlled by large avalanches, in which the CDW phase in localized regions moves forward abruptly in response to a small change in the field. Since the collapse to universality above threshold was understood in terms of the jerky dynamics, the same should be true even below threshold. This is indeed found to be the case numerically. Thus even below threshold, we seek to expand around the mean field solution for linear cusped potentials.

Before proceeding with the calculations, it is necessary to consider a possibility that might make this procedure erroneous. While we have answered the question of history dependence by choosing a specific approach to threshold, namely increasing $F$ monotonically, this does not completely eliminate problems that arise from the existence of many metastable states. An expansion around the mean field solution of the type we develop here corresponds to first increasing the force within an infinite ranged model, and then tuning the elastic coupling to 
a short ranged form (i.e., restoring the $\nabla^{2} \phi$ term in Eq.(5) which was replaced by $\langle\phi\rangle-\phi$ in mean field theory). On the other hand, the physically relevant critical behaviour corresponds to first tuning the elastic coupling to a short ranged form, and then increasing the force within a short ranged model. When there are many metastable solutions, as is the case below threshold, the two methods could in principle yield different critical behaviour.

Since the CDW phase at any site moves only forward when the force is raised monotonically, such problems from metastability could arise if reducing the range of the elastic interactions from a mean field theory were to result in the phases at different sites trying to move backwards. While this will of course be true for some of the phases, for which the coupling to their neighbours tends to retard their motion, we shall see later in this paper that the polarizability exponent $\gamma$ is in fact larger for $d<4$ than in mean field theory. Thus the dominant effect of reducing the range of the elastic interactions is to make the phases move forward, making the polarization (and hence the polarizability) more divergent than in mean field theory. We therefore expect our procedure of expanding around the mean field solution to yield the correct critical behaviour.

The expansion around mean field theory is carried out following the prescription of Sompolinsky and Zippelius, 19 the details are given in Ref. 9. The method yields an impurity averaged generating functional for the correlation and response functions of the phase $\phi$, of the form

$$
\begin{aligned}
\bar{Z}=\int[d \Phi] & {[d \hat{\Phi}] \exp \left\{-\int d^{d} x d t \hat{\Phi}(x, t)\left[\partial_{t}-\nabla^{2}+r\right] \Phi(x, t)+\hat{\Phi}(x, t)\left[F-F_{M F}(\langle\phi(t)\rangle)\right]\right.} \\
& \left.-\int d^{d} x d t_{1} d t_{2} \hat{\Phi}\left(x, t_{1}\right) \hat{\Phi}\left(x, t_{2}\right) C\left[\left\langle\phi\left(t_{1}\right)\right\rangle+\Phi\left(x, t_{1}\right)-\left\langle\phi\left(t_{2}\right)\right\rangle-\Phi\left(x, t_{2}\right)\right]\right\}
\end{aligned}
$$

Here $\Phi$ and $\hat{\Phi}$ are dummy field variables, in terms of which the long wavelength low frequency forms of the truncated correlation and response functions of $\phi$ are given by

$$
\begin{aligned}
\partial^{n}\left\langle\phi\left(x_{1}, t_{1}\right) \ldots \phi\left(x_{m}, t_{m}\right)\right\rangle_{\text {trunc }} / \partial \varepsilon\left(x_{m+1}, t_{m+1}\right) \ldots \partial \varepsilon\left(x_{m+n}, t_{m+n}\right) \\
=\left\langle\Phi\left(x_{1}, t_{1}\right) \ldots \Phi\left(x_{m}, t_{m}\right) \hat{\Phi}\left(x_{m+1}, t_{m+1}\right) \hat{\Phi}\left(x_{m+n}, t_{m+n}\right)\right\rangle
\end{aligned}
$$

where the left hand side represents the generalized response to a perturbing force $\varepsilon(x, t)$ 
added to the right hand side of Eq.(5), and the right hand side is the expectation value using the measure of Eq.(8). $\Phi(x, t)$ is like a coarse grained version of the phase $\phi(x, t)$. It gives the deviations of $\phi(x, t)$ from its spatially averaged value, $\langle\phi\rangle$, and must satisfy the consistency condition $\langle\Phi\rangle=0$, which determines $F-F_{M F}$. Various other terms, that do not affect the critical behaviour, have been suppressed in Eq.(8).

Eq.(8) is similar to the generating functional obtained in Ref. 9 above threshold, except for new terms that we discuss in the next paragraph. The function $C$ is the same as the mean field phase phase correlations as a function of time above threshold: $C\left(v t_{1}-v t_{2}\right)=$ $\left\langle\left[\phi\left(t_{1}\right)-v t_{1}\right]\left[\phi\left(t_{2}\right)-v t_{2}\right]\right\rangle$. Below threshold, the spatial average of the phase, $\langle\phi(t)\rangle$, replaces $v t$. For any value of $\langle\phi\rangle$, the function $F_{M F}(\langle\phi\rangle)$ is the force that would result in an average displacement $\langle\phi\rangle$ in the mean field approximation to Eq.(5), while the function $F$ is the force that would produce the same average displacement in the full short-ranged model of Eq.(5). As mentioned in the previous paragraph, the difference between these two, which is the coefficient of the second term in Eq.(8), can be found by the consistency condition $\langle\Phi\rangle=0$. This again is similar to the case above threshold.

Despite the similarities, there are important differences between Eq.(8) and the corresponding generating functional above threshold $\mathrm{B}$. There is a non-zero 'mass term' in Eq.(\$), $r \hat{\Phi} \Phi$, which results in a finite response of $\phi$ to a spatially uniform dc force. Above threshold, where this term vanishes exactly due to time translational invariance of the system, $\chi(q=0, \omega)$ has a $1 / i \omega$ singularity, corresponding to a finite response in the velocity of $\phi$. The bare value of $r$ is the inverse of the mean field polarizability $\chi^{\uparrow}(F)$ (along the monotonically increasing path); $r$ vanishes at threshold, although the manner in which it approaches zero at $F_{T}$ depends on the distribution $\rho(h)$ of the pinning strengths. The generating functional $\bar{Z}$ also differs from the form above threshold by various other terms that also vanish at threshold like $\hat{\Phi} r \Phi$ does. For instance, the coefficient of the second term, $F-F_{M F}(\langle\phi\rangle)$ has corrections of $O\left(\left[F_{T}-F\right]^{2}\right)$. These, however, do not affect the critical behaviour and are not shown in Eq.(8). Also, it is only when the mean field correlation and response functions are evaluated within the monotonically increasing approach to threshold that we obtain the 
functional forms in Eq.(8) that resemble those above threshold. For other (non-monotonic) approaches, this will not be the case. Above threshold, where the steady state behaviour is unique, no particular path needs to be specified.

The variable $\langle\phi(t)\rangle$ in the arguments of the functions $F_{M F}$ and $C$ in Eq.(\&) implicitly controls the dependence on $F$. It is necessary to increase $\langle\phi(t)\rangle$ adiabatically in order for the form in Eq.(8) to be valid; otherwise, there would be various transients that would have to be included in the equation. Since the increase of the force is much slower than all the dynamics of the $\mathrm{CDW}$, the equal time response and correlation functions really give the static behaviour. Although it would seem that Eqs.(8) and (9) can be used to obtain responses to time dependent perturbations, there is an implicit constraint that the perturbing force must satisfy for our entire approach to be valid: $\varepsilon\left(t_{1}\right) \geq \varepsilon\left(t_{2}\right)$ for $t_{1} \geq t_{2}$. This constraint prevents us from obtaining the ac response, $\chi(\omega, F)$.

We now carry out a renormalization group analysis of Eq.(8) along the lines of Ref. 9. We rescale space and time as $x=b x^{\prime}$ and $t=b^{z} t^{\prime}$, and integrate out modes of all frequencies in the momentum shell between the (rescaled) momenta $b \Lambda$ and $\Lambda$, where $\Lambda$ is the upper cutoff in momentum. The rescaling of the fields $\Phi$ and $\hat{\Phi}$ are fixed in mean field theory by the requirement that all quadratic terms in the exponential have to be invariant under this transformation, except the $\hat{\Phi} r \Phi$ term; the variation of this last term is interpreted as a change in $F$ under renormalization. Fixing the other quadratic terms in the exponential yields $\hat{\Phi}=b^{-z-d / 2} \hat{\Phi}^{\prime}$ and $\Phi=b^{z-d / 2} \Phi^{\prime}$, with the dynamic exponent $z$ fixed at 2 by comparing the scaling of the $\partial_{t}$ and $\nabla^{2}$ terms. The consistency of the mean field scaling is verified by ascertaining that all higher order operators are irrelevant; this is indeed the case for $d>4$, which is therefore the upper critical dimension. This upper critical dimension is the same as for $F>F_{T}$.

At $d=4$, the entire function $C\left[\left\langle\phi\left(t_{1}\right)\right\rangle+\Phi\left(t_{1}\right)-\left(t_{1} \leftrightarrow t_{2}\right)\right]$ becomes marginal. For $d<4$, the critical behaviour is changed from its mean field form. As discussed in Ref. 9, for $d<4$ the function $C$ splits up into a constant part $C_{s}$ that controls the static distortions in the phase, and a functional operator $C(\phi)$ that controls the dynamics. The two of these decouple 
from each other, and scale differently. For the dynamics above threshold, where the steady state solution is periodic, it is necessary to preserve the invariance under the transformation $\phi \rightarrow \phi+2 \pi$. For $d<d_{c}$, the upper critical dimension, fluctuations in $\phi$ scale in the same way as $\langle\phi\rangle=v t$; this then requires $\Phi$ to be invariant under rescaling, as discussed in Ref. 9. The operator whose coefficient is the constant part of $C, C_{s}$, is then relevant, leading to anomalous scaling of the static correlations.

Below threshold, it is precisely these static correlations that we are interested in. Since the mean phase $\langle\phi\rangle$ reaches a constant value for any fixed value of $F<F_{T}$ in steady state (with $\langle\phi\rangle$ increasing with $F$ ), the periodicity of the phase variable need not be preserved under the rescaling of the renormalization group. Accordingly, we choose a scaling for the fields $\hat{\Phi}$ and $\Phi$ different from that of Ref. 9 :

$$
\hat{\Phi}=b^{-d / 2-z} \hat{\Phi}^{\prime}, \quad \Phi=b^{2-d / 2} \Phi^{\prime} .
$$

The scaling of the $\hat{\Phi}$ field is fixed by requiring the invariance of the $C_{s}$ term, which is unrenormalized by loop corrections. (This is because all loop terms involve differences between the function $C$ at two different values of its argument, which are not changed by an addition of a constant $C_{s}$. Although it is conceivable that singular corrections could affect this result, we assume here that this is not the case.) As discussed in Ref. 9, any renormalization of the $\hat{\Phi} \Phi$ term from the operator $C$ (as well as from higher order irrelevant operators) must be of the form $\hat{\Phi} \partial_{t} \Phi$, and thus the $\hat{\Phi} \nabla^{2} \Phi$ term also has no loop corrections, fixing the scaling of $\Phi$. The scaling of the $r \hat{\Phi} \Phi$ term yields a renormalized polarizability $\chi^{\prime}=\chi / b^{2}$. Finally, the $\left(F-F_{M F}\right) \hat{\Phi}$ term, which does receive loop corrections, is relevant, so that the scaling of $F$ can also be obtained by power counting: $\left(F-F_{T}\right)^{\prime}=b^{d / 2}\left(F-F_{T}\right)$, thus yielding the correlation length exponent

$$
\nu=2 / d
$$

and, from the scaling of $\chi$,

$$
\gamma=4 / d
$$


as mentioned in Eqs. (11) and (2)).

Although it is possible to obtain these exponents directly from a very simplified model of CDWs 11 , the exponent z cannot be obtained from a similar analysis and, as we shall now see, is nontrivial. A more detailed discussion of Ref. 11 is given in Section V. The exponent $z$ is not affected by the difference between the scaling here and that of Ref. 9, although under the scaling used here, the operator $C$ is dangerously irrelevant, flowing towards a singular function of zero amplitude, with constant second derivative $C^{\prime \prime}(\phi)$. (With the scaling of Ref. 9, $C$ flows towards a regular function of constant amplitude, with the same second derivative.) The $O(\epsilon)$ result for $z$, obtained in Ref. 9, depends on the second derivative of $C$, and is given by

$$
z=z\left(F>F_{T}\right)=2-\epsilon / 3+O\left(\epsilon^{2}\right) .
$$

The breakdown of two sided scaling for CDWs, with $\nu=2 / d$ below threshold, and $\nu=1 / 2$ above threshold, is now seen as the result of the different static and dynamic behaviour of the system, rather than being due to any fundamental difference between the properties above and below threshold. Even above threshold, the static correlation length still exists, and was indirectly obtained in Ref. 9 as the length that controls the finite size scaling behaviour. The static correlation function above threshold varies as a simple power law with distance, and does not show any crossover at the static (or dynamic) correlation length, so that the static correlation length cannot be obtained directly.

\section{NUMERICAL RESULTS}

We now compare these results with those from numerical simulations. Previous simulations have often been carried out with the continuous variable dynamics of Eq.(5), with $x$ discretized to a lattice. However, it is possible to obtain much more accurate results with automaton models, which may be viewed as a singular limit of Eq.(5), in which the pinning potential at any site has very narrow and steep wells, so that $\phi(x ; t)=\beta(x)+2 \pi m(x ; t)$, 
with $m$ an integer variable. A further approximation is made by discretizing time, 20 the dynamics of $m(x ; t)$ is then

$$
\begin{aligned}
m(x ; t) & =m(x ; t-1)+1 & & \text { if } F+\nabla^{2}[2 \pi m(x ; t-1)+\beta(x)]+h(x)>0 \\
& =m(x ; t-1) & & \text { if } F+\nabla^{2}[2 \pi m(x ; t-1)+\beta(x)]+h(x) \leq 0 .
\end{aligned}
$$

Here the $\nabla^{2}$ operator is the discrete Laplacian. This automaton model (and variants of it) are numerically much more efficient than direct simulations of Eq.(5). Since for small $d$, the critical behaviour is independent of the form of the pinning potential used, we shall rely mostly on the automaton model simulations in this section, referring to continuous dynamics only for comparison.

It is more convenient to express the dynamics in terms of a local "curvature" variable

$$
c(x)=\sum_{y \in\langle x y\rangle}[m(y)-m(x)]+\operatorname{Int}\left[\left\{F+\nabla^{2} \beta(x)+h(x)\right\} / 2 \pi\right]
$$

where the sum over $y$ is restricted to sites neighbouring $x$. The first term in this equation is the discretized form of $\nabla^{2} m$. By construction, $c(x)$ is an integer valued variable. From Eq.(14), we see that the dynamics can be expressed in terms of $c(x)$ as

$$
\begin{aligned}
& c(x) \rightarrow c(x)-n \\
& c(y) \rightarrow c(y)+1 \forall y \in\langle x y\rangle \quad \text { if } c(x)>0 .
\end{aligned}
$$

Here $n$ is the number of neighbours that the site $x$ has. We can view this as a sandpile model 12 if we treat $c(x)$ as the height of sand at the site $x$; when this exceeds a preset threshold (zero in Eq.(16)) one grain of sand falls off from $x$ to each of its neighbouring sites. As $F$ is increased, $\operatorname{Int}\left[\left(F+\nabla^{2} \beta(x)+h(x)\right) / 2 \pi\right]$ increases by 1 whenever its argument crosses an integer. From Eq.(15), we see that increasing $F$ towards threshold is equivalent to dropping grains of sand randomly on different sites of the system. There is, however, a slight difference in the manner in which sand is added as compared to standard sandpile models: since the randomness from $\nabla^{2} \beta(x)+h(x)$ is quenched, sand grains are dropped in a random order on the different sites of the lattice one by one, with no site being revisited 
twice in a cycle. When every site has been visited exactly once, the whole cycle is repeated in the same order. In contrast to this, in standard sandpile models the addition of each sand grain is an independent random process, and there are no correlations between the sites chosen. For a sufficiently large system, where the repeating cycle is very long, it is reasonable to expect the difference between cyclic and uncorrelated addition of sand grains to be inconsequential.21

Another difference between CDWs and usual sandpiles is that open boundary conditions are normally used for sandpiles, whereas periodic boundary conditions are used for CDWs. With periodic boundary conditions, a grain of sand that exits the system at one boundary reenters at the opposite boundary. Again, when the system size is much larger than the characteristic avalanche size, this difference should not affect the behaviour.22.23 In this section, we shall use numerical results from CDW and sandpile models, both with periodic boundary conditions, to compare with our analytical results. Comparisons with earlier simulation 13,14 on sandpiles with open boundary conditions will be discussed in subsequent sections.

Figure 1 shows a finite size scaling plot for the polarization $P(F, L)$ (measured from the initial state $m=0$ at $F=0$ ) for $d=2$ as a function of the driving force $F$ and the linear size of the system, $L$. The finite size scaling of the polarization should be of the form

$$
P=|f|^{-\gamma+1} \hat{P}\left(L|f|^{\nu}\right)
$$

where $f$ is the reduced force, $F / F_{T}-1$. The collapse to a scaling form in the numerical data shown in Figure 1 is not very good. This is probably due to the fact that the polarization in any state involves the polarizability integrated from the initial to the present state. Corrections to scaling might thus be expected to persist in the polarization until the system is very close to threshold. Figure 2 shows a similar scaling plot for the polarizability, obtained by a numerical derivative of the polarization. The data here scale better when $L|f|^{\nu}$ is large, but show a large scatter very close to threshold due to numerical uncertainties. Figure 3 shows a scaling plot for the polarization in the sandpile version of the dynamics of Eq.(14); 
the scaling form works much better here. From the data in Figure 3, we obtain estimates for the critical exponents: $\gamma(d=2)=1.98 \pm 0.03$ and $\nu(d=2)=0.98 \pm 0.03$, in agreement with Eqs.(12) and (11).

Simulations with the continuous dynamics of Eq.(5) have larger numerical uncertainties. As detailed in Ref. 5, a log-log plot of the polarization as a function of $f$ appears to yield an exponent $\gamma=1.8 \pm 0.15$, although the slight upward curvature seen as $F \rightarrow F_{T}$ allows for the possibility that the asymptotic value of $\gamma$ is indeed 2, as given by Eq.(12). The collapse of the data to a single scaling function is also not very satisfactory, but can be used to obtain the correlation length exponent $\nu$ as $1.0 \pm 0.1$. A more accurate value for $\nu$ is obtained from the width of the distribution of threshold fields $F_{T}$ for various systems of size $L$ with different realizations of randomness, since one expects finite size effects to become important when the characteristic size of an avalanche is of the order of the size of the system. Fitting $\Delta F_{T}$ to the form $L^{-\nu}$ yields $\nu=1.01 \pm 0.03$.

For three dimensional systems, the numerical uncertainties in the behaviour of the polarizability are larger. Here we show only the results for simulations on the sandpile model (with periodic boundary conditions) for which, as in two dimensions, the scaling is better. Figure 4 shows a scaling plot of the polarizability, from which we obtain $\gamma=1.31 \pm 0.08$ and $\nu=0.68 \pm 0.04$. Since it is possible to determine the location of the threshold field very accurately, one can also plot the polarization at threshold as a function of system size. As shown in Figure 5, $P_{s}\left(F_{T}, L\right)$ appears to scale as $L^{0.57}$, consistent with Eqs. (11) and (12) for $d=3$. (The error bars shown in Figure 4 are from the statistical scatter in the data; owing to the small range in $L$, this is too conservative an estimate for the true uncertainties.) The exponent $\nu$ can be obtained independently from the scatter in the threshold field to be $\nu=0.68 \pm 0.02$, as shown in Figure 6 .

From the duration of an avalanche as a function of its size, we obtain the dynamic exponent $z$. The size $s$ of an avalanche, defined as the total number of sites participating in it, scales as $l^{d}$, where $l$ is its linear extent, while $z$ is defined through $t(l) \sim l^{z}$, so that $t(s) \sim s^{z / d}$. Figures 7(a) and 7(b) are $\log -\log$ plots of $t(s)$ versus $s$ for CDWs in 2 and 3 dimensions 
respectively. From these we obtain $z(d=2)=1.32 \pm 0.04$ and $z(d=3)=1.65 \pm 0.06$. This agrees very well with our result $z\left(F<F_{T}\right)=z\left(F>F_{T}\right) ; z\left(F>F_{T}\right)$ can be found from the velocity exponent $\beta$ through $z\left(F>F_{T}\right)=2 \beta$, and $\beta$ is known independently from numerical simulations above threshold to be $\beta(d=2)=0.64 \pm 0.03$ and $\beta(d=3)=0.81 \pm 0.036$ 目

\section{DISTRIBUTION OF AVALANCHES}

We now turn to the distribution of avalanches of different sizes in the critical region. As discussed earlier, there are differences in the forms for the continuous variable and automaton models. This is because the total increase in the phase $\phi$ at any site is bounded above by $2 \pi$ in the continuous variable models. No such constraint exists for automata, for which several avalanches of roughly the same size are grouped together into a single large avalanche. This may lead to different number distributions for avalanches in the continuous and automaton models. We first deal with the avalanche distribution for automaton models, returning later in this section to continuous variable models.

We conjecture a scaling form for the number distribution of avalanchest:

$$
n(s ; f) d s=\frac{1}{s^{\kappa / d}} \hat{n}\left(s|f|^{\nu d}\right) \frac{d s}{s}
$$

where $n(s ; f) d s d f$ is the total number of avalanches of size between $s$ and $s+d s$ that occur in a unit volume of the system when the reduced force $\left(F / F_{T}-1\right)$ is changed from $f$ to $f+d f$. This equation defines the exponent $\kappa$. (Here we have assumed that the same $\nu$ is associated with the characteristic size of the avalanches as well as the finite size effects.)

We define the moment of an avalanche as the total change in polarization that results from

it. This is related to the change in the phase at every site in the avalanche by $\delta P=\sum_{i=1}^{s}\left(\delta \phi_{i}\right)$. For continuous dynamics, where $\delta \phi_{i} \leq 2 \pi$ for all the sites, the moment scales as the size of the avalanche. For automaton models, the two are related by a nontrivial exponent:

$$
\delta P \sim s^{\Gamma}
$$


Equations (18) and (19) can be used to obtain an exponent identity relating $\kappa$ and $\Gamma$ to $\gamma$. The total change in polarization upon changing the force by $d f$ is found by integrating Eq.(18) to be of the form $\Delta P \sim f^{(\kappa-\Gamma d) \nu} d f$. Comparing with Eq.(7), we obtain

$$
\gamma=(\Gamma d-\kappa) \nu
$$

Substituting Eqs.(12) and (11) in this equation yields

$$
\Gamma d-\kappa=2 .
$$

Figure 8(a) shows a finite size scaling plot of the distribution of avalanches for the automaton model in two dimensions. By fitting to the form of Eq.(18), we obtain

$$
\kappa(d=2)=0.36 \pm 0.03
$$

Figure $8(\mathrm{~b})$ shows a similar plot for $d=3$, from which we can obtain

$$
\kappa(d=3)=1.00 \pm 0.06
$$

The values of $\nu$ that are obtained from these scaling plots are $\nu(d=2)=0.98 \pm 0.03$ and $\nu(d=3)=0.68 \pm 0.03$, consistent with Eq.(11) (as well as the scatter in $\left.F_{T}\right)$. The scaling of the moment of an avalanche with its size can be used to obtain numerically

$$
\Gamma(d=2)=1.15 \pm 0.05
$$

and

$$
\Gamma(d=3)=1.00 \pm 0.02
$$

which, together with the numerical values for $\kappa$, are consistent with Eq.(21). In the limit as $F \rightarrow F_{T}$, a power law distribution for the avalanches is obtained. If $\hat{n}(x)$ is not a singular function of its argument $x$ for small $x$, the limiting form of the distribution can be used to obtain $\kappa$ and $\Gamma$. If, however, $\hat{n}(x)$ scales as $x^{(\alpha-1) / 2 d \nu}$ for small $x$, the apparent power law in the avalanche distribution would change from $\kappa$ to $\kappa-(\alpha-1) / 2 \nu$, while leaving $\Gamma$ unchanged. 
A singular form for $\hat{n}(0)$ would imply that the total number of avalanches generated by a small increase in $F$ (which is dominated by small avalanches) would be singular as $F_{T}$ is approached; we have verified numerically that this is not the case for the automaton models.

It is interesting to note that Eq.(21) has been derived earlier by Majumdar and Dhar 10 for sandpile models, by a completely different method that relies only on properties of clusters at the critical point 25 Since sandpiles at the critical point are current driven, $J=0^{+}$, instead of being tuned by a driving force $F$, any prefactor in $n(s)$ that is singular in $f$ would not be seen in the scaling. The fact that Eq.(21) and the result of Majumdar and Dhar are identical can thus be taken as a proof that $\hat{n}(0)$ is not singular. Our results in Eqs. (22) through (25) are also consistent with numerical simulations on sandpile models in two and three dimensions. 13

As mentioned in the previous section, the dynamic exponent $z$ can be found numerically from the scaling of the duration of an avalanche with its size; the results in both two and three dimensions are fairly close to a first order truncation of the $\epsilon$-expansion result of Eq.(13). Even at the critical point, the duration of the avalanches can be used to obtain $z$; this allows us to compare with the result for two-dimensional sandpilest 1 that $z=5 / 4$, which agrees with our numerical results. For three dimensions, there is no exact calculation of $z$ at present to compare our numerical results with. However, previous simulations on (a transformed version of the sandpile model with open boundary conditions 26 have yielded $z(d=3)=1.62 \pm 0.01$, in agreement with our results.

For continuous variable models, we have seen that the bound on the change in phase at any site in an avalanche constrains $\Gamma_{c}$ to being 1 . (The subscript $c$ here denotes continuous variable dynamics.) From Eq.(21), we then obtain $\kappa_{c}=d-2$, so that both the exponents involved in the avalanche distribution are determined.27 This result is only true if the driving force is increased infinitesimally. In practice, the force is increased in small steps; no matter how small the step size, one presumably eventually crosses over into a regime where successive avalanches at the same site trigger within a single force step, and the exponents change to those of the automaton models. Although the analytical treatment of Section III 
is applicable only for continuous variable dynamics, a proper analysis of the singular part of the non-linear response to finite increases in $F$ might yield the automaton values for the exponent $\Gamma$; we have so far been unable to carry this out. Note that the numerical results in Eqs.(22) through (25) are not very far from the trivial values $\Gamma=1$ and $\kappa=d-2$; in fact, in three dimensions they are consistent to within the numerical uncertainties.

Instead of triggering avalanches by increasing the driving force, it is possible to do so by applying small random 'kicks' to the phases at different sites, which may be considered to mimic the effect of thermal noise. Remarkably, for small thermal noise, the characteristic linear extent of an avalanche still scales with the distance from the zero temperature threshold force, but with a different correlation length exponent:

$$
\xi_{t h} \sim\left(F_{T}-F\right)^{-\nu_{t h}}
$$

with $\nu_{t h} \neq \nu$. The 'thermal' correlation length exponent has been found numerically by Myers to be $\nu_{t h} \approx 0.50$ in two dimensions. As shown in Figure 9, the same result is true for $d=3$ as well. A proper understanding of this phenomenon is still lacking; however, it is possible that considerations about the equilibrium behaviour (at finite temperature) in the absence of a driving force may be applicable here. At low temperatures, one might expect the presence of a driving force to produce an overall forward drift of the CDW, while still preserving the equilibrium properties locally. In equilibrium, the dynamics can be expressed in terms of correlations in the pinning potential. This is in contrast to our zero temperature analysis, where the physically relevant correlation function $C$ is related to force-force correlations. 6 The potential-potential correlation function is related to the function $C$ integrated twice; as shown in Ref. 29, in equilibrium it is necessary for this to be periodic in its argument. Changing $C$ by a constant $C_{s}$ is therefore no longer allowed in equilibrium. Setting $C_{s}$ to zero implies that the static correlations no longer scale differently from the dynamic correlations, and $\nu_{t h}=1 / 2$. Further work on this, especially on crossovers from equilibrium to nonequilibrium properties, is clearly required. 


\section{DISCUSSION}

Most of the work on sandpile models has been on the properties at the critical point. However, Tang and Bak 14 have considered sandpile models away from the critical point; through a combination of scaling arguments and numerical simulations, they have obtained results similar to some of ours. For instance, by considering the diffusive nature by which sand grains propagate through the system, they obtain the exponent identity $\gamma / \nu=2$, which is the same as from Eqs.(12) and (11). With the assumption that the distribution of avalanches is independent of system size for sufficiently large systems, and the implicit assumption that the moment of an avalanche is proportional to its size, they obtain an exponent identity connecting $\gamma$ and $\kappa$, which is a special case of the general result derived in the previous section. The first of these two assumptions is equivalent to $\hat{n}(0)$ not being singular; we have seen in the previous section that this is correct, both by comparing our scaling law to that of Majumdar and Dhar 15 and by direct numerical verification. The second assumption, that $\Gamma=1$, is not correct, even though it is approximately valid for $d=2$ which is the case Tang and Bak focus on (and actually much better for $d=3$ ). A few other results that they obtain are for the behaviour of transients, and cannot be compared with our work. The authors have also noted that if the critical behaviour in these nonequilibrium models were to be like that of equilibrium statistical mechanical systems, various exponent identities relating $\beta$ to other quantities would result. These exponent identities can be compared with our results, and are found not to be valid.

Surprisingly, the value obtained by them for $\nu$ through numerical simulations is $\nu(d=$ $2)=0.7$, which disagrees completely with our analytical and numerical result $\nu(d=2)=1$. It is not clear what this difference is due to, although a similar value of $\nu(\nu=0.76)$ has apparently been observed in other simulations as well in which the sandpile is perturbed

in a manner different from ours30. As discussed in the previous section, with different types of kicks on the sandpile (that may or may not have physically meaningful analogs in the continuous variable models) $\nu$ can be very different; this might be the source of the 
discrepancy. Our simulations on sandpile models, detailed in the previous section, yield $\nu(d=2) \approx 1$.

A related problem to CDWs, that of the depinning of interfaces in random media, has been analyzed in the moving phase by methods similar to those used for CDWs.31,28 Unlike the case for CDWs, the statics and dynamics do not behave differently; physically, this is because as the interface moves forward it experiences different regions of impurities and is thus unable to build up anomalous static correlations. Formally, this is seen by the absence of an unstable direction at the fixed point of $C$ when the appropriate boundary conditions are taken. Since this separate scaling of static and dynamic correlations is the source of the difference between $\nu\left(F>F_{T}\right)$ and $\nu\left(F<F_{T}\right)$ for CDWs, two-sided scaling will be valid for interfaces. The relation $\gamma / \nu=2$ can be verified to be still true by the procedure in this paper.

Parisi and Pietronero1 11 have considered a model for CDWs which is equivalent to choosing the pinning potentials $V(\phi)$ at different sites to be of the form $V(\phi ; x)=$ $h(x) \phi-2 \pi h(x) \sum_{n} \theta(\phi-\beta(x)-2 \pi n)$, so that the phase at any site experiences a constant retarding force from the pinning potential, equal to $-h(x)$. In addition to this, there is the elastic force from the neighbouring sites, and the external driving force. (Although they have a $\theta$-function constraint that prevents the total force at any site from being negative, we have seen that when threshold is approached monotonically the phases at all the sites always move forward, so that this constraint is inconsequential for the critical behaviour.) Although this model correctly yields $\gamma=4 / d$ and $\nu=2 / d$, the dynamic exponent $z$ is wrong, being trivially equal to 2 . The reason for this partially correct result can now be understood: after averaging over the randomness, their model corresponds to the one we have considered here, with the dynamic part of the correlation function, $C(\phi)$, set to zero, but with $C_{s} \neq 0$. This does constitute a fixed point, at which loop corrections from $C(\phi)$ are absent, so that $z=2$. However, this fixed point is unstable to the one that we have analyzed in this paper, so that the model that they have considered constitutes a pathological limit for pinning potentials. In particular, it is possible to replace the vertical drop in $V(\phi)$ at $\phi=\beta+2 \pi n$ with a 
very steep section of non-zero width. The critical behaviour of this model has been found numerically $\mathrm{\theta}$ to the same as for the other models that we have considered here, although the width of the critical region above threshold can be seen through a perturbation expansion to vanish as the width of the steep sections grows smaller. 32

As mentioned in Section III, due to the restriction that the driving force must be monotonically increasing in time, it is not possible to obtain the ac response $\chi(\omega, F)$ from our analysis. It is not clear at present if some variation of our approach may prove adequate for this purpose. It is important to remember that the universality that exists for various different models with regard to the monotonic approach to threshold is considerably limited for $\chi(\omega, F)$ even in low dimensions.

In this paper, we have analyzed the critical behaviour of charge density waves as the depinning threshold is approached monotonically from below. Our success in this was due to the fact that, for this special path to threshold, the existence of many metastable states was argued not to invalidate the perturbation expansion we carry out. Although for any particular problem one must consider afresh whether such a perturbation expansion is valid, it may be possible to use similar techniques for other systems.

\section{Acknowledgements}

We would like to thank Daniel Fisher, Satya Majumdar and Chao Tang for useful discussions. O.N. is supported by a Junior Fellowship from the Society of Fellows at Harvard University, and wishes to acknowledge the hospitality of the Institute for Theoretical Physics in Santa Barbara where part of this work has been completed. 


\section{REFERENCES}

1 D.S. Fisher, Phys. Rev. Lett. 50, 1486 (1983) and Phys. Rev. B31, 1396 (1985).

2 This is only correct if one neglects various effects like thermal fluctuations and dislocations; in real materials, these either round out the sharp phase transition or drive it first order. [See, e.g., S.N. Coppersmith, Phys. Rev. Lett. 65, 1044 (1990), Phys. Rev. B44, 2887 (1991); S.N. Coppersmith and A.J. Millis, Phys. Rev. B44, 7799 (1991).]

3 P.B. Littlewood, Phys. Rev. B33, 6694 (1986); H. Matsukawa, J. Phys. Soc. Jpn. 57, 3463 (1988); S.N. Coppersmith and D.S. Fisher, Phys. Rev. A38, 6338 (1988).

4 P. Sibani and P.B. Littlewood, Phys. Rev. Lett. 64, 1305 (1990).

5 A.A. Middleton and D.S. Fisher, Phys. Rev. Lett. 66, 92 (1991) and Phys. Rev. B47, 3530 (1993).

6 A.A. Middleton, Ph.D. thesis, Princeton University.

7 C.R. Myers, Ph.D. thesis, Cornell University; C.R. Myers and J.P. Sethna, (unpublished).

8 A.A. Middleton, unpublished; see also A.A. Middleton, O. Biham, P.B. Littlewood and P. Sibani, Phys. Rev. Lett. 68, 1586 (1992).

9 O. Narayan and D.S. Fisher, Phys. Rev. Lett. 68, 3615 (1992) and Phys. Rev. B 46, 11520 (1992).

${ }^{10}$ A.A. Middleton, Phys. Rev. Lett. 68, 670 (1992).

${ }^{11}$ G. Parisi and L. Pietronero, Europhys. Lett. 16, 321 (1991).

${ }^{12}$ P. Bak, C. Tang and K. Wiesenfeld, Phys. Rev. Lett. 59, 381 (1987).

${ }^{13}$ S.S. Manna, Physica A179, 249 (1991).

${ }^{14}$ C. Tang and P. Bak, Phys. Rev. Lett. 60, 2347 (1988).

${ }^{15}$ S. Majumdar and D. Dhar, Physica A185, 129 (1992). 
${ }^{16}$ We thank Daniel Fisher for pointing this out to us.

${ }^{17}$ H. Fukuyama and P.A. Lee, Phys. Rev. B17, 535 (1978); P.A. Lee and T.M. Rice, Phys. Rev. B19, 3970 (1979); L. Sneddon, M.C. Cross and D.S. Fisher, Phys. Rev. Lett. 49, 292 (1982).

18 The velocity exponent is often called $\zeta$ in previous references.

${ }^{19}$ H. Sompolinsky and A. Zippelius, Phys. Rev. B25, 6860 (1982); A. Zippelius, Phys. Rev. B29, 2717 (1984).

20 The discretization of time in unit steps results in a constant force being indistinguishable from an ac force whose frequency is an integer. This gives rise to mode-locking in the sliding state, as discussed in Ref. 8. However, the discreteness of time does not affect the statics.

${ }^{21}$ This is the case for generic initial conditions for $c(x)$. For instance, if the initial configuration is chosen to be $m=0$, the second term in Eq.(15) results in $c(x)$ being random. However, if we choose $c(x)$ to be identically zero initially, the behaviour with cyclic addition of sand grains is atypical.

${ }^{22}$ For $F>F_{T}$, where an infinite avalanche runs across the entire system, the different boundary conditions give rise to very different behaviour. The phase transition to a moving state that is seen with periodic boundary conditions is destroyed with open boundary conditions, where the system expels an appropriate number of sand grains to bring it back to its critical point.

${ }^{23}$ Note that both the quenched randomness in the addition of sand grains as well as the different boundary conditions imposed result in different finite size corrections for CDWs than for sandpiles, even below threshold.

${ }^{24}$ We assume here that the avalanche clusters are compact. 
25 The notation they use is slightly different from ours: they use exponents $\tau_{d}=1+[\kappa-(\alpha-$ 1) $/ 2 \nu] / d$ and $\tau_{s}=1+\left(\tau_{d}-1\right) / \Gamma$. Using the result from Eq.(11) that $\nu=2 / d$, their result agrees with Eq.(21) only if $\alpha=1$. (Although their result is expressed for the specific case of $d=2$, it can be generalized straightforwardly.)

${ }^{26}$ A.J. Guttmann and R.J. Bursill, J. Stat. Phys. 59, 1 (1990). See S.N. Majumdar, Phys. Rev. Lett. 68, 2329 (1992) for the mapping to sandpiles.

${ }^{27}$ Note that $\alpha_{c}$ is not equal to 1 ; thus the number of avalanches at a fixed value of $f$ scales with sizes with the exponent $\left[\kappa_{c}-\left(\alpha_{c}-1\right) / 2 \nu\right] / d=-\left(\alpha_{c}-1\right) / 2 d \nu$. It is this exponent that is denoted as $\kappa$ in Ref. 5 .

${ }^{28}$ O. Narayan and D.S. Fisher (unpublished).

${ }^{29}$ T. Giamarchi and P. LeDoussal (unpublished).

${ }^{30}$ J. Carlson, E.R. Grannan, C. Singh and G.H. Swindle (unpublished).

31 T. Nattermann, S. Stepanow, L.-H. Tang and H. Leschhorn, J. Phys. II (France) 2, 1483 $(1992)$

${ }^{32}$ O. Narayan (unpublished). 


\section{FIGURES}

FIG. 1. Attempted finite-size scaling of the polarization $P$ for the CDW automaton in $d=2$ with best fits $\gamma \approx 2$ and $\nu \approx 1$. No set of exponents gives a single curve, due to the large finite size effects and the arbitrary constant in the definition of the polarization. Symbols indicate the size of the system, while the numbers in parentheses indicate the number of samples averaged over.

FIG. 2. Scaling of the polarizability $\chi^{\uparrow}$ (numerical derivative of the data in Fig. 1) in the $d=2$ CDW automaton model with fitted exponents $\gamma=2 \pm 0.15$ and $\nu=1 \pm 0.1$. The scatter at low scaled fields $L|f|^{\nu}$ is due to statistical fluctuations.

FIG. 3. Scaling of the polarization in the $d=2$ sandpile automaton model. The scaling collapse is much better than in the $d=2 \mathrm{CDW}$ model. From the range of exponents for which the scaling collapse is within finite-size errors and statistical uncertainties, we find $\gamma=1.98 \pm 0.03$ and $\nu=0.98 \pm 0.03$, in agreement with the analytical results in the text.

FIG. 4. Scaling of the polarizability in the $d=3$ sandpile automaton model. From the range of exponents for which the scaling collapse is within finite-size errors and statistical uncertainties, we find $\gamma=1.31 \pm 0.08$ and $\nu=0.68 \pm 0.04$, in agreement with the analytical results of the text.

FIG. 5. Polarization at threshold for the $d=3$ sandpile automaton with periodic boundary conditions as a function of linear size $L$. Error bars represent $1 \sigma$ statistical uncertainties. The fitted slope in the figure indicates only statistical errors and are an underestimate of the true uncertainty in the ratio $(\gamma-1) / \nu$. From such data, we estimate $(\gamma-1) / \nu=0.57 \pm 0.08$.

FIG. 6. Relative sample-to-sample fluctuations $\Delta F_{T} / F_{T}$ in the threshold field $F_{T}$ as a function of linear size for the $d=3$ sandpile model with periodic boundary conditions. From the fitted slope, the finite-size correlation length exponent $0.68 \pm 0.02$ is obtained. 
FIG. 7. Numerical derivatives of the logarithm of the duration $t$ of an avalanche with respect to the logarithm of the volume $s$, for (a) the $d=2$ CDW model and (b) the $d=3$ sandpile model. The approach to a constant at large $s$ gives the scaling $t \sim s^{z / d}$, with $z=1.32 \pm 0.04$ in $d=2$ and $z=1.65 \pm 0.06$ in $d=3$.

FIG. 8. Scaled avalanche distributions seen at different fields for (a) 100 samples of size $512^{2}$ in the $d=2$ CDW model and (b) 110 samples of size $128^{3}$ in the $d=3$ sandpile model. Avalanches result from the (adiabatically) increasing field. We find $\kappa=0.36 \pm 0.03$ and $\nu=0.98 \pm 0.03$ for $d=2$ and $\kappa=1.00 \pm 0.06$ and $\nu=0.68 \pm 0.03$ for $d=3$.

FIG. 9. Scaled distribution of avalanches for the $d=3$ sandpile automaton model at fixed field (total height). Avalanches are in response to individual "thermal kicks" which increase the phase at one location (by redistributing the curvature variable). The scaling collapse is within errors for $\kappa=0.99 \pm 0.03$ and $\nu=0.50 \pm 0.02$ 


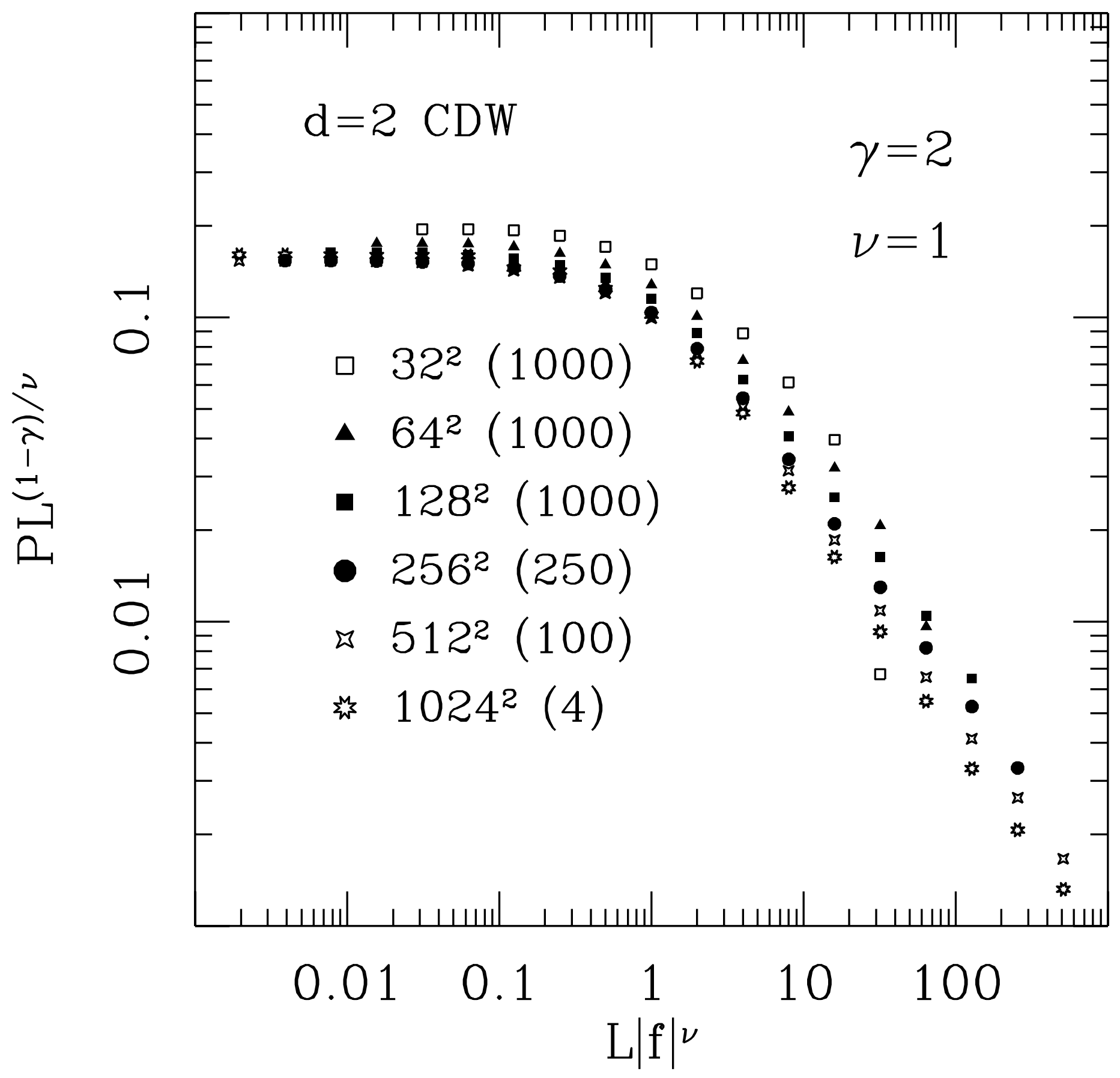

Fig. 1 - Narayan and Middleton 


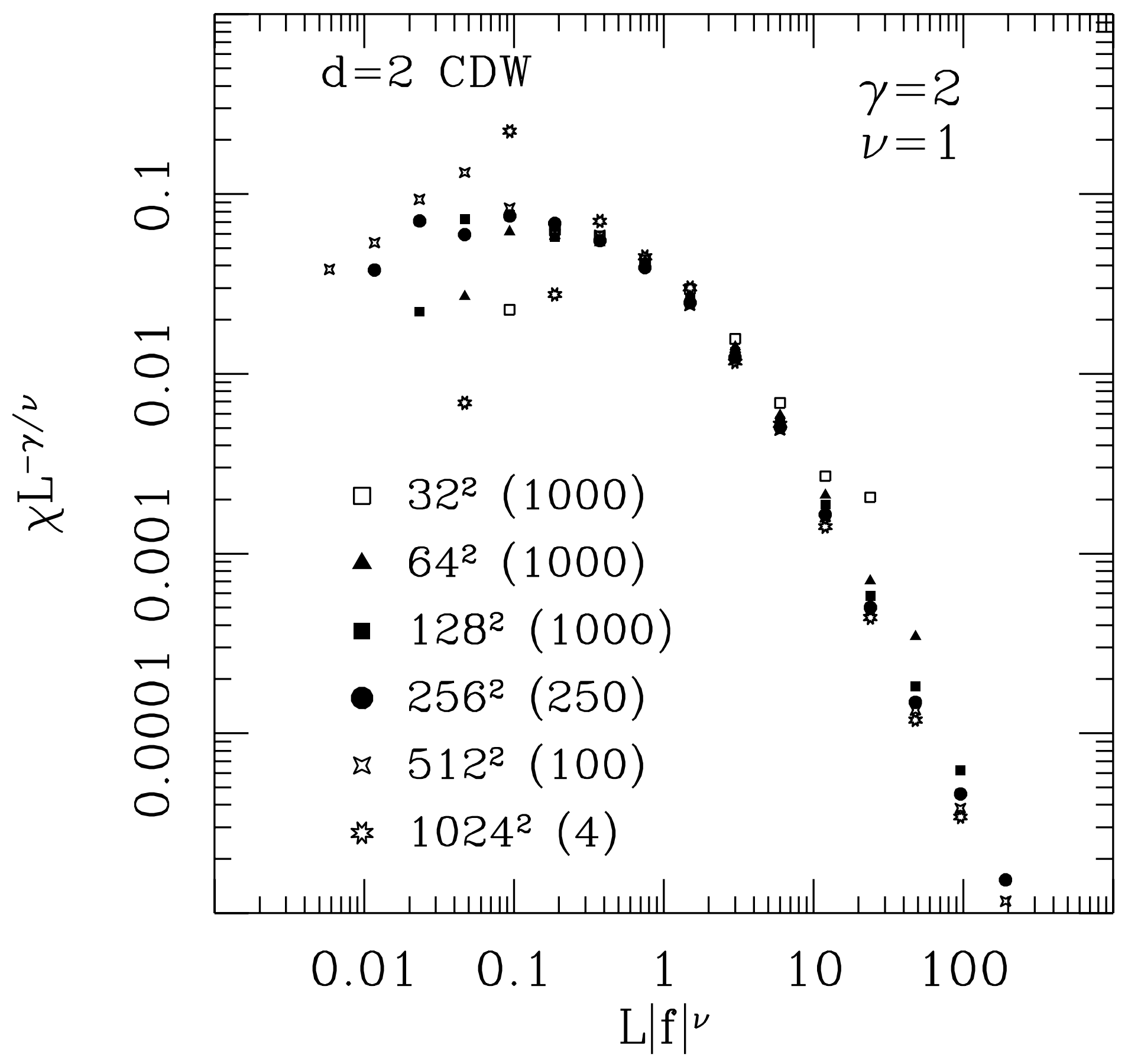

Fig. 2 - Narayan and Middleton 


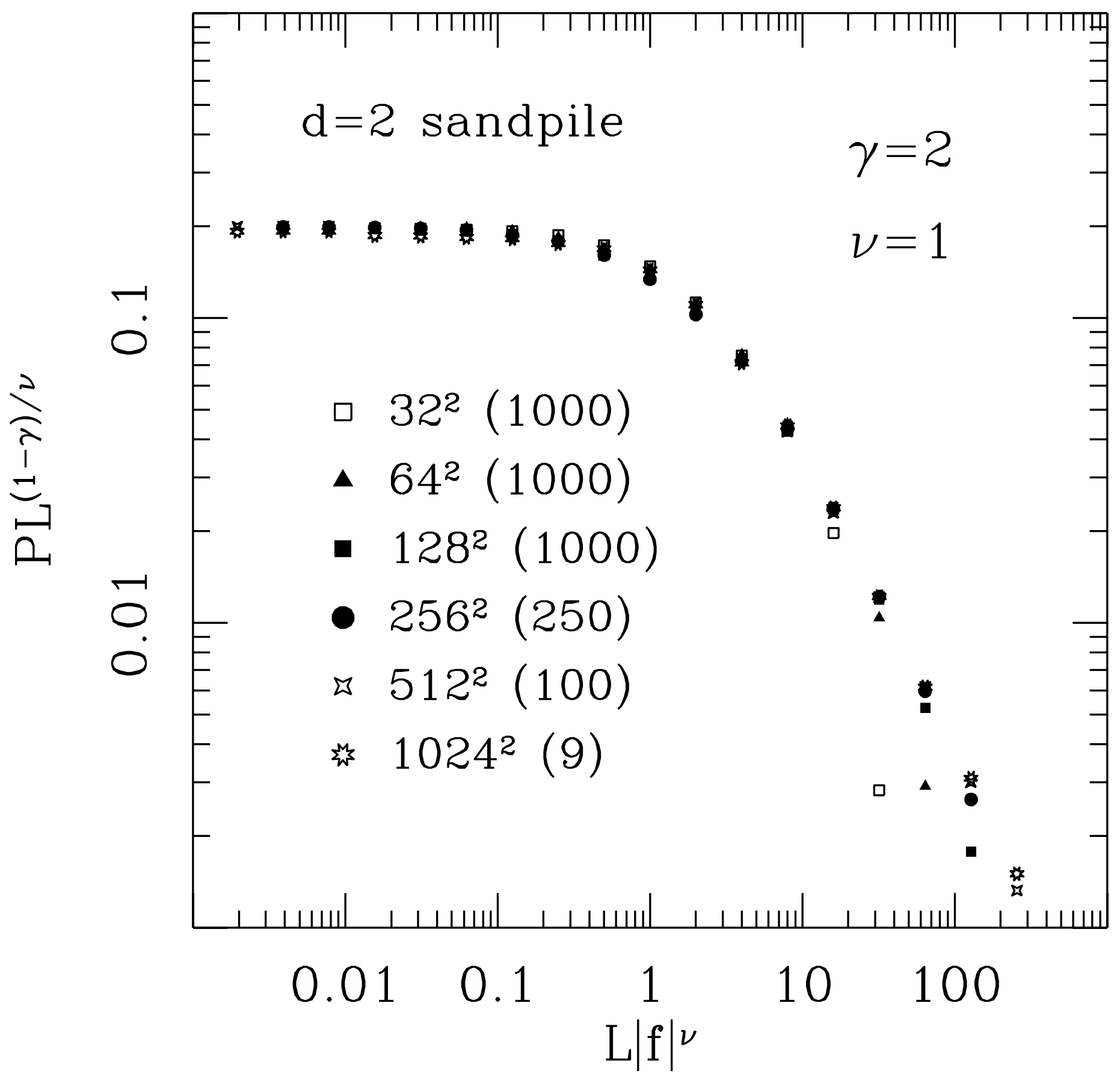

Fig. 3 - Narayan and Middleton 


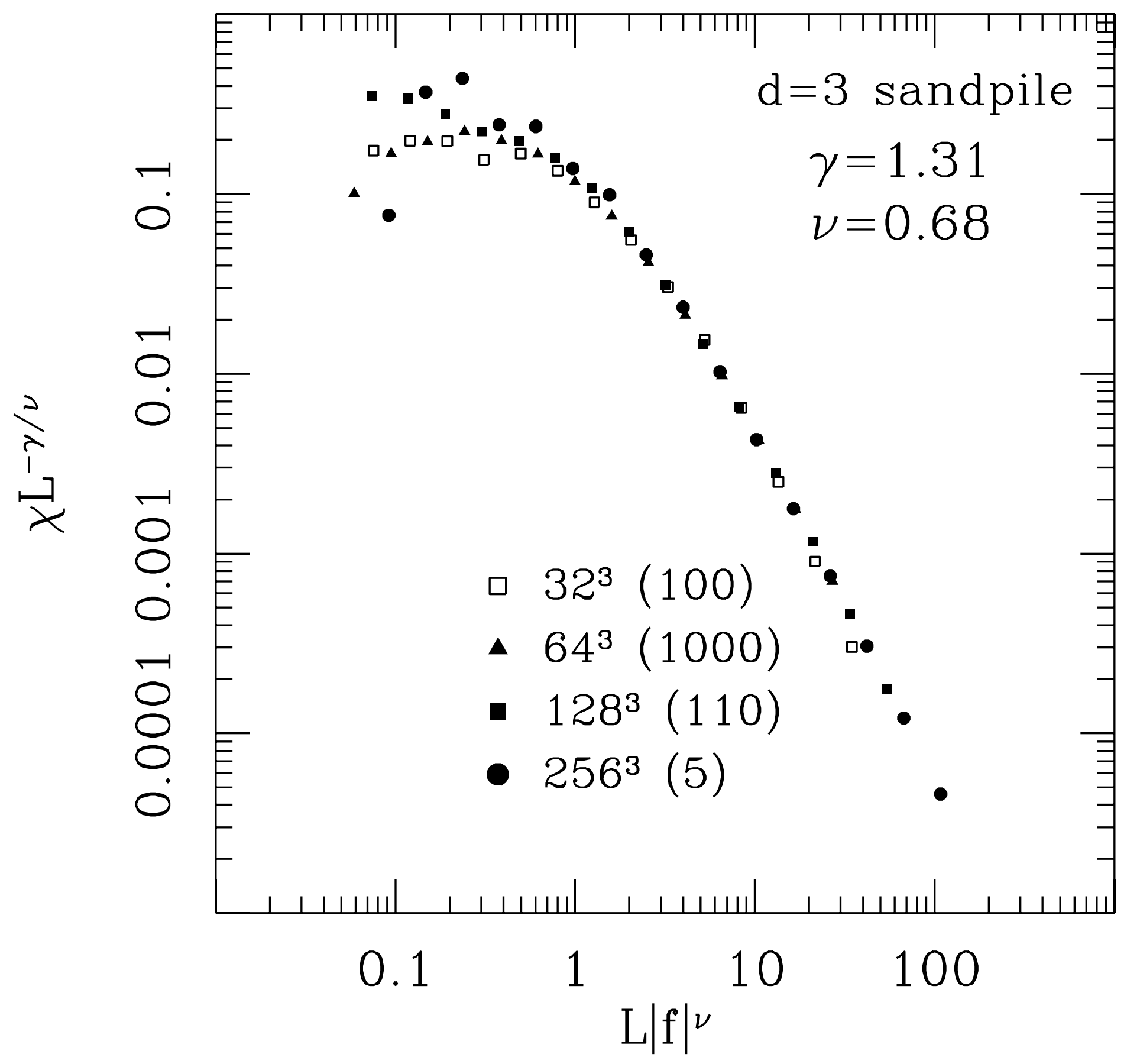

Fig. 4 - Narayan and Middleton 


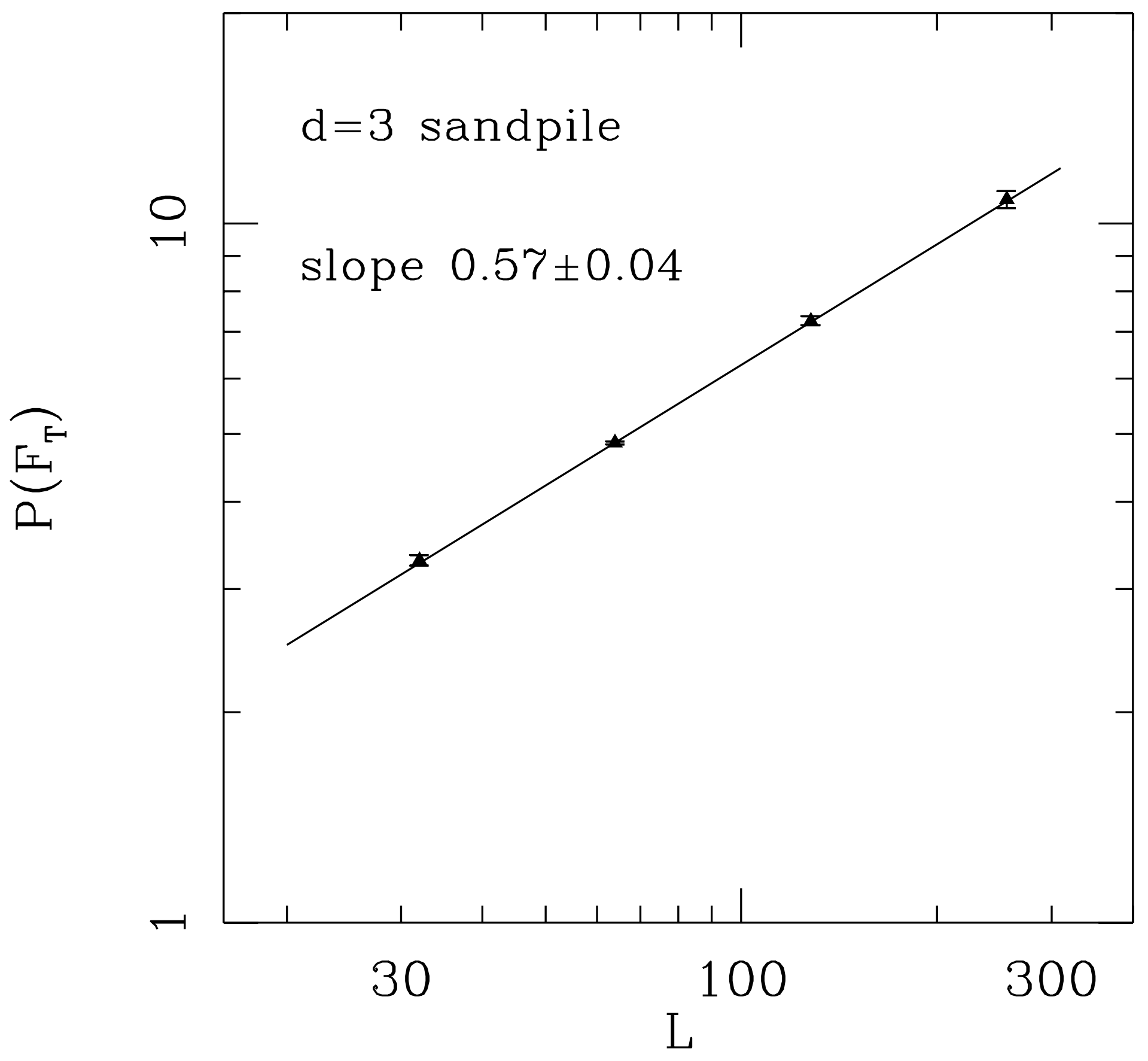

Fig. 5 - Narayan and Middleton 


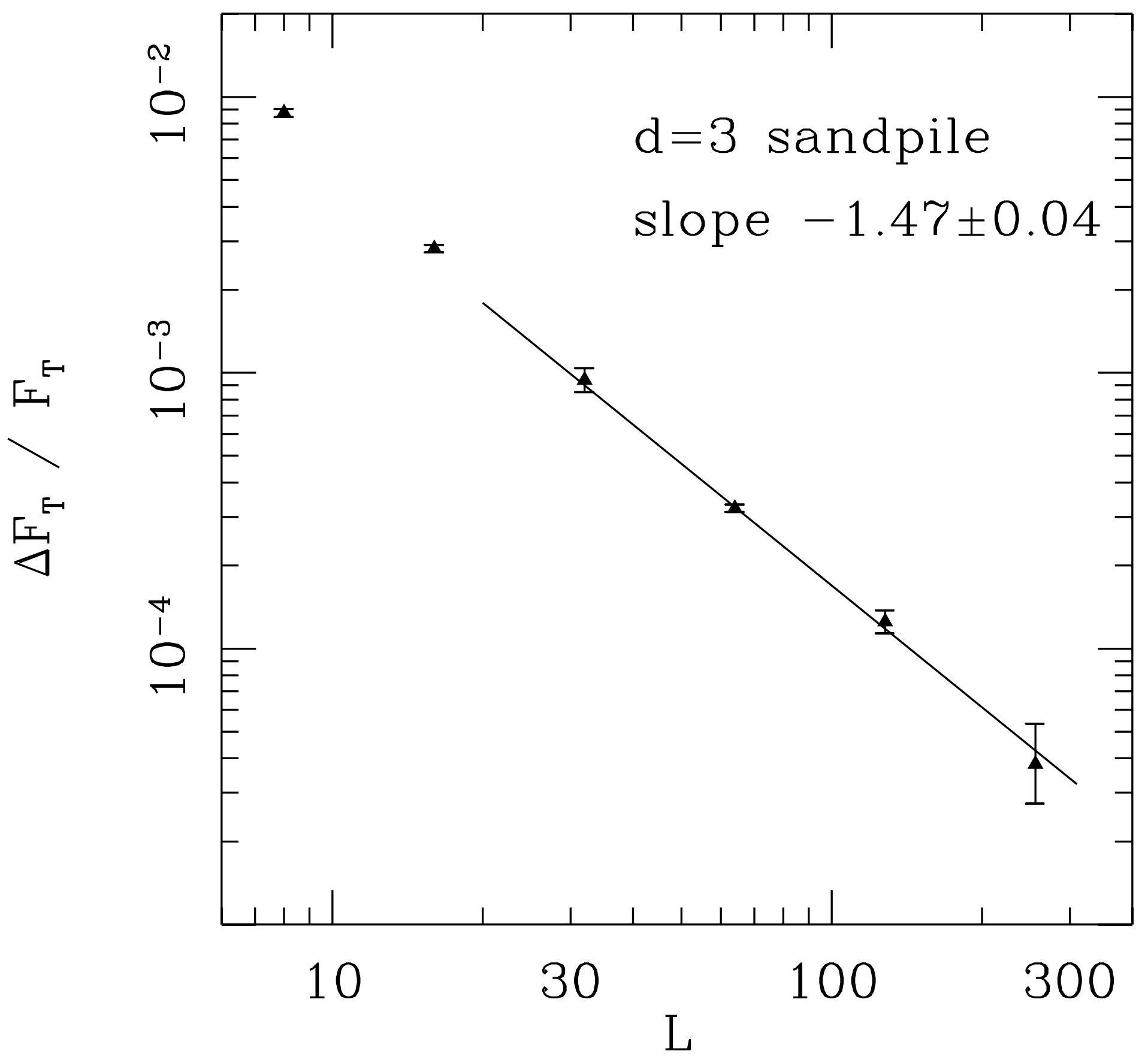

Fig. 6 - Narayan and Middleton 


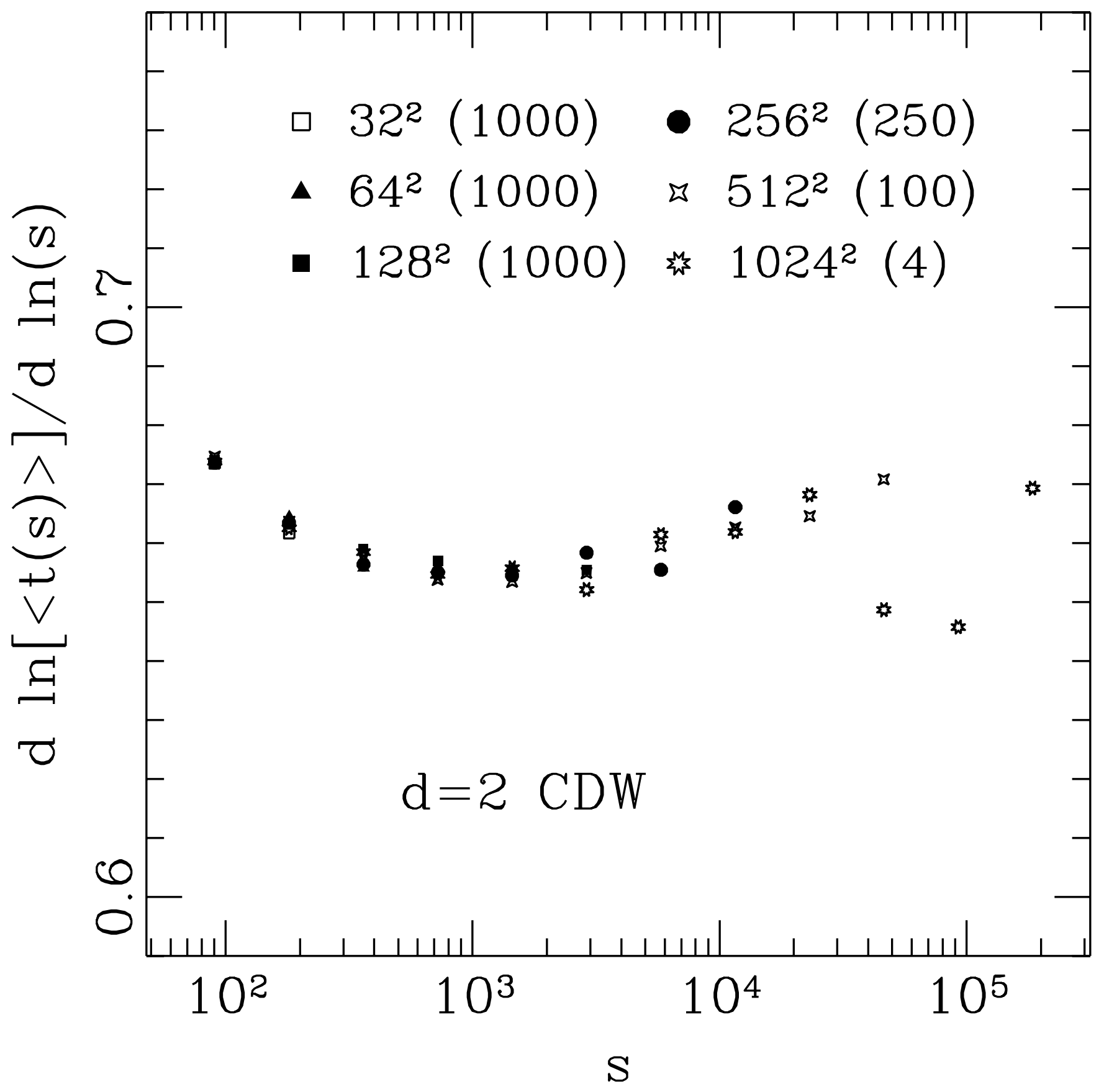

Fig. 7a - Narayan and Middleton 


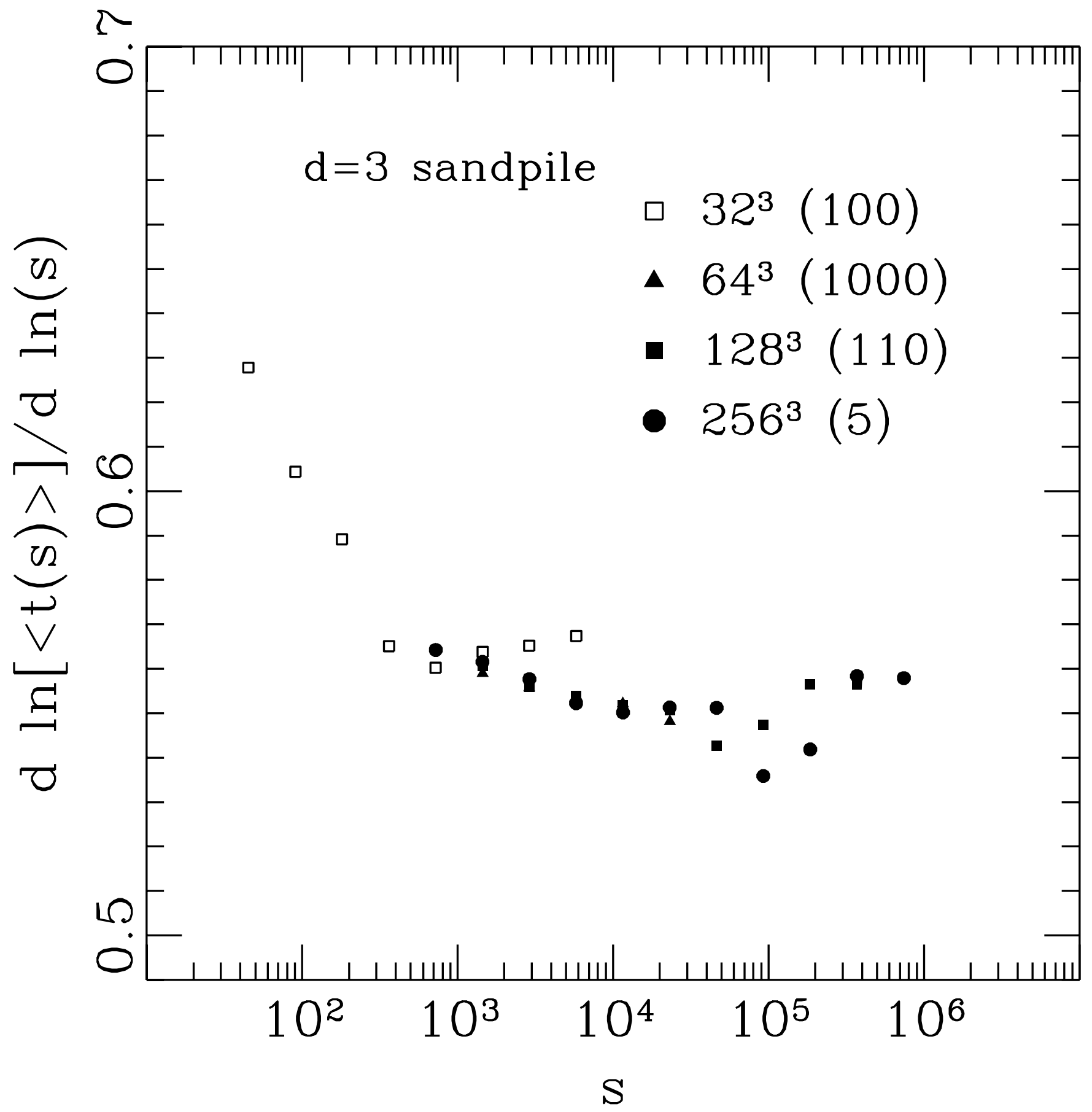

Fig. 7b - Narayan and Middleton 


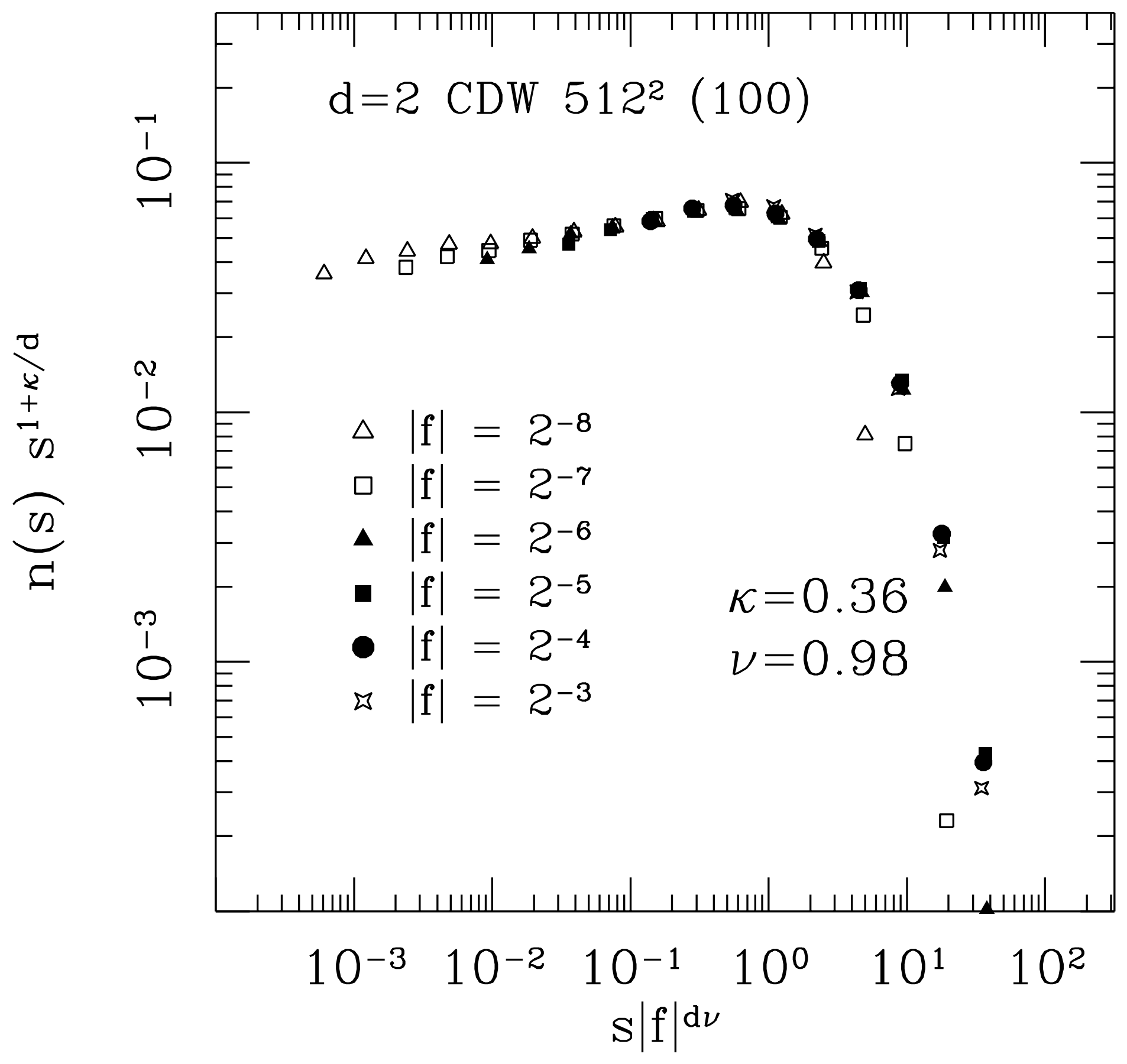

Fig. 8a - Narayan and Middleton 


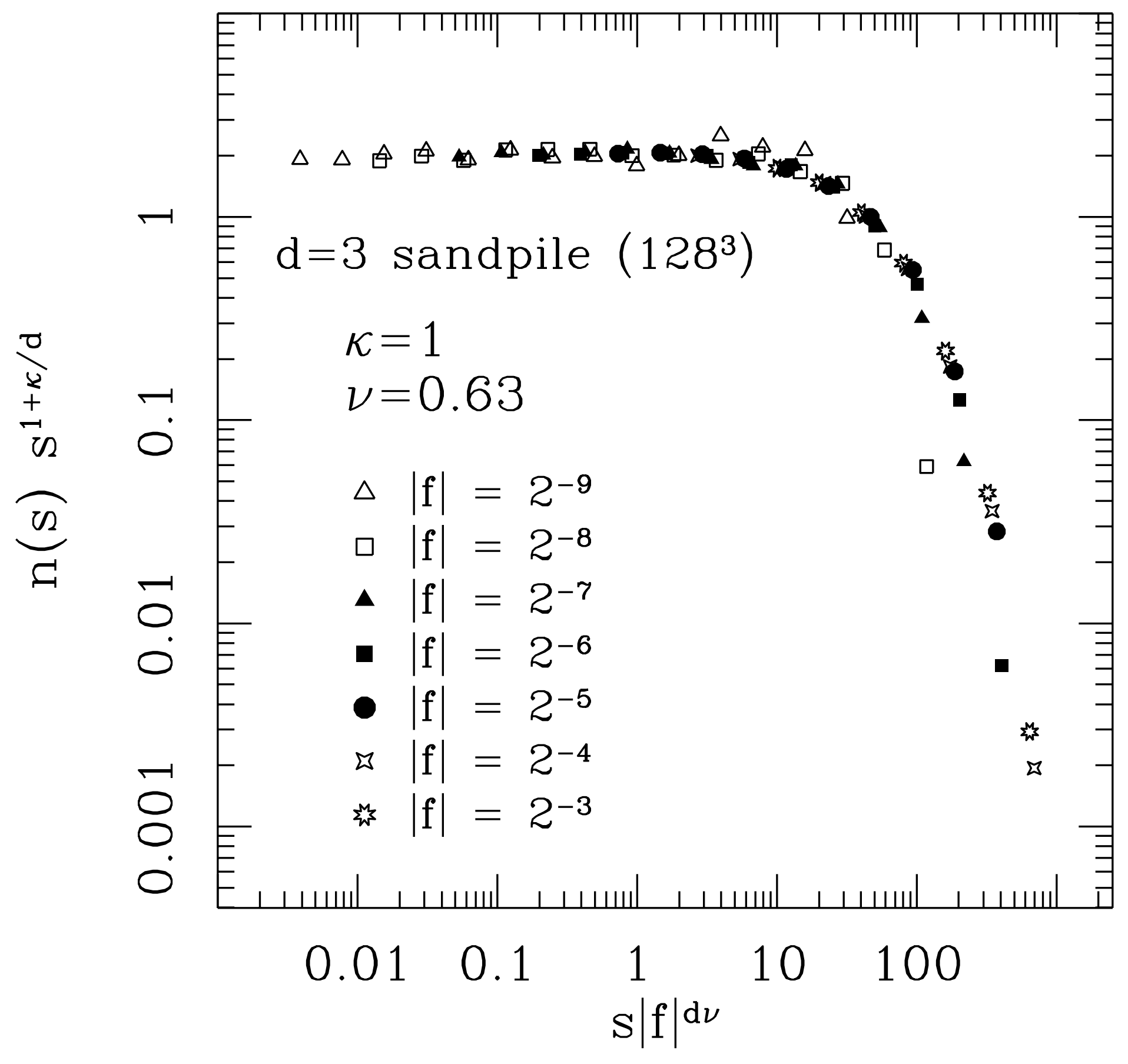

Fig. $8 \mathrm{~b}$ - Narayan and Middleton 


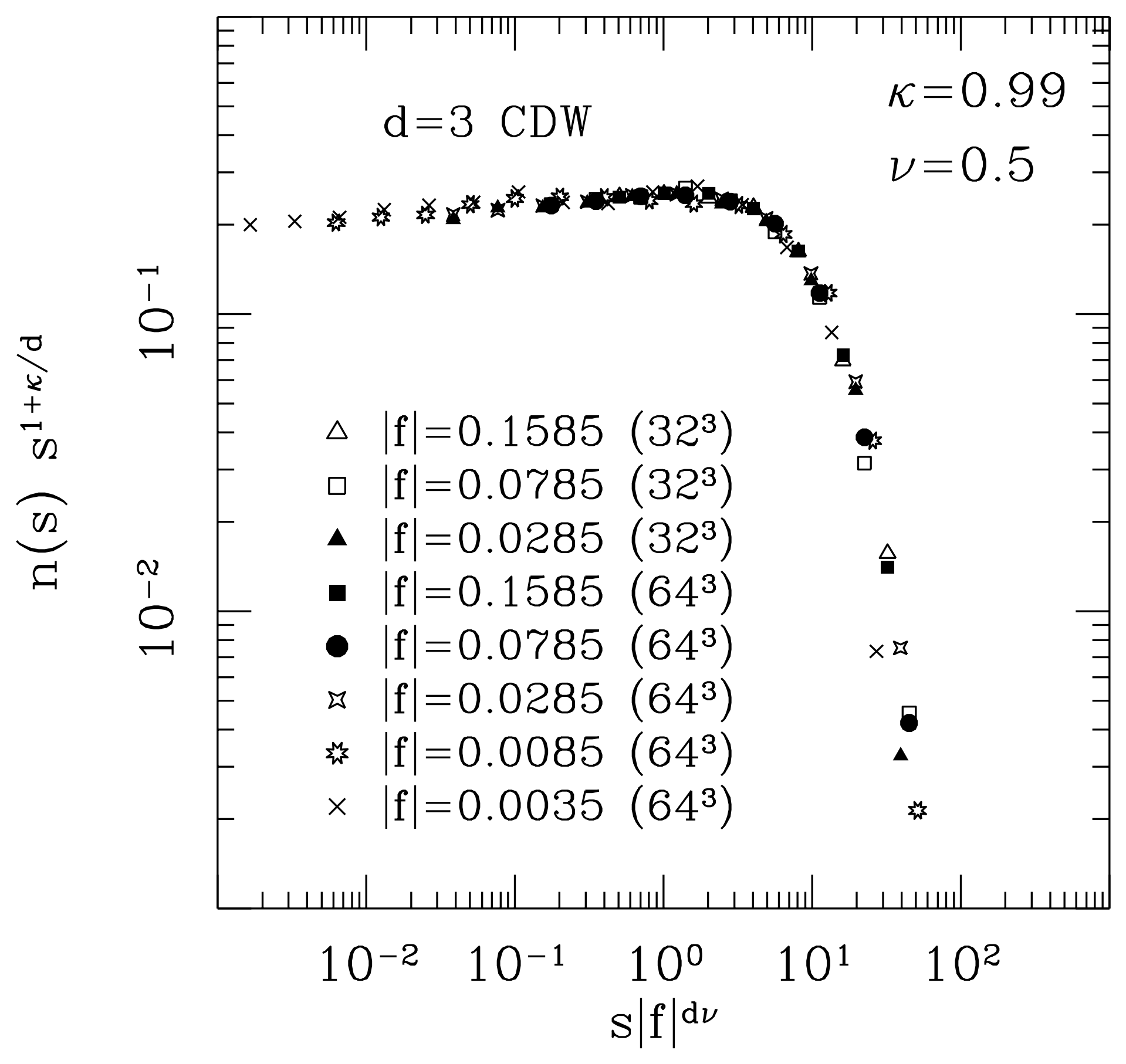

Fig. 9 - Narayan and Middleton 